International Journal of Applied Mathematics

Volume 33 No. $1 \quad 2020,157-170$

ISSN: 1311-1728 (printed version); ISSN: 1314-8060 (on-line version)

doi: http://dx.doi.org/10.12732/ijam.v33i1.12

\title{
INVERSE BOUNDARY-VALUE PROBLEM FOR LINEARIZED EQUATION OF MOTION OF A HOMOGENEOUS ELASTIC BEAM
}

\author{
Kh.E. Abbasova ${ }^{1}$, Y.T. Mehraliyev ${ }^{2}$, E.I. Azizbayov ${ }^{3} \S$ \\ ${ }^{1}$ Department of Digital Economy and ICT \\ Azerbaijan State University of Economics \\ Baku, AZ1001, AZERBAIJAN \\ 2 Department of Differential and Integral Equations \\ Baku State University \\ Baku, AZ1148, AZERBAIJAN \\ ${ }^{3}$ Department of Computational Mathematics \\ Baku State University \\ Baku, AZ1148, AZERBAIJAN
}

\begin{abstract}
The present paper is devoted to the study of classical solution of an inverse boundary-value problem for the linearized equation of motion of a homogeneous elastic beam with an over-determination condition. The goal of the work is to determine both solution and the unknown coefficient together for the considered problem in the rectangular region. First, in order to investigate of solvability of the inverse problem, we reduce original problem to the auxiliary problem with trivial data. Applying the Fourier method and contraction mappings principle, the existence and uniqueness of the classical solution of the obtained equivalent problem is proved. Furthermore, using the equivalence, the unique solvability of the appropriate auxiliary inverse problem is shown.
\end{abstract}

AMS Subject Classification: 35L25, 35Q74, 35R30, 35A01, 35A02, 35A09 Key Words: inverse boundary-value problem; homogenous beam; classical solution; Fourier's method

Received: November 6, 2019

(c) 2020 Academic Publications

${ }^{\S}$ Correspondence author 


\section{Introduction and Preliminaries}

In the rectangular region $D_{T}: 0 \leq x \leq 1,0 \leq t \leq T$ we consider inverse boundary-value problem for the one-dimensional equation of motion of a homogeneous elastic beam [15]

$$
\begin{aligned}
u_{t t}(x, t)+u_{x x x x}(x, t) & +\beta u_{x x}(x, t) \\
& =p(t) u(x, t)+f(x, t), \quad(x, t) \in D_{T},
\end{aligned}
$$

for the unknown functions $u(x, t), p(t)$, with conditions

$$
\begin{gathered}
u(x, 0)=\varphi(x), u_{t}(x, 0)=\psi(x), 0 \leq x \leq 1, \\
u(0, t)=u(1, t)=u_{x x}(0, t)=u_{x x}(1, t)=0,0 \leq t \leq T, \\
\int_{0}^{1} g(x) u(x, t) d x=h(t), 0 \leq t \leq T,
\end{gathered}
$$

where $T, \beta$ are positive integers, $f(x, t), \varphi(x), \psi(x), g(x)$, and $h(t)$ are known functions of $x \in[0,1]$ and $t \in[0, T]$.

In many cases the practical requirements lead to the problems of determining the coefficients or the right hand side of a differential equation (ordinary or in partial derivatives) from some known functional of its solution. Such problems are called inverse problems of mathematical physics. The practical importance of inverse problems is so great (they arise in various fields of human activity, such as seismology, mineral exploration, biology, medicine, desalination of seawater, movement of liquid in a porous medium, etc.) which puts them a series of the most actual problems of modern mathematics. The presence in the inverse problems of additional unknown functions requires that in the complement to the boundary conditions that are natural for a particular class of differential equations, impose some additional conditions - overdetermination conditions. The basics of the theory and practice of studying inverse problems of mathematical physics were established and developed in the fundamental works of the outstanding scientists A.N. Tikhonov [23], M.M. Lavrent'ev [16], V.K. Ivanov [11], and their followers.

Inverse problems associated with differential equations of various types, have been studied by many papers and monographs, in particular $[1,3,4,6,7$, $9,10,13,14,17,19,20]$. But the problem statement and the proof techniques used in this paper are different from those presented in these works. The technique used in this paper is based on the passing from the original inverse 
problem to the new equivalent one, the study of the solvability of the equivalent problem, and then in the reverse transition to the original problem.

Moreover, the vibrations and wave movements of an elastic beam were investigated by B.S. Bardin [5], T.P. Goy [8], D.V. Kostin [15], Y.T. Mehraliyev [2], Yu.A. Mitropolsky [18], J.M. Thompson [22], V.Z. Vlasov [24], and et al.

The simplest nonlinear model of the motion of a homogeneous beam is described by the equation

$$
\frac{\partial^{2} w}{\partial t^{2}}+\frac{\partial^{4} w}{\partial x^{4}}+k \frac{\partial^{2} w}{\partial x^{2}}+\alpha w+w^{3}=0
$$

where $w$ is beam deflection (after the displacements of the points of the midline of the elastic beam located along the $x$-axis). Note that a similar equation also arises in the theory of crystals, in which $w$ is parameter of order [12].

We introduce the set of functions

$$
\tilde{C}^{2,4}\left(D_{T}\right)=\left\{u(x, t): u(x, t) \in C^{2}\left(D_{T}\right), u_{x x x x}(x, t) \in C\left(D_{T}\right)\right\} .
$$

Definition 1. The pair $\{u(x, t), p(t)\}$ defined in the domain $D_{T}$ is said to be a classical solution of the problem (1)-(4), if the functions $u(x, t) \in \tilde{C}^{2,4}\left(D_{T}\right)$ and $p(t) \in C[0, T]$ satisfies Eq. (1), condition (2) on $[0,1]$, and the statements (3)-(4) on the interval $[0, T]$.

Theorem 2. Suppose that $f(x, t) \in C\left(D_{T}\right), \varphi(x), \psi(x) \in C[0,1], g(x) \in$ $L_{2}(0,1), h(t) \in C^{2}[0, T]$, and the conditions

$$
\int_{0}^{1} g(x) \varphi(x) d x=h(0), \int_{0}^{1} g(x) \psi(x) d x=h^{\prime}(0)
$$

hold. Then the problem of finding a classical solution of (1)-(4) is equivalent to the problem of determining the functions $u(x, t) \in \tilde{C}^{2,4}\left(D_{T}\right)$ and $p(t) \in C[0, T]$ from the (1)-(3), and satisfying the condition

$$
\begin{aligned}
h^{\prime \prime}(t)+ & \int_{0}^{1} g(x) u_{x x x x}(x, t) d x+\beta \int_{0}^{1} g(x) u_{x x}(x, t) d x \\
& =p(t) h(t)+\int_{0}^{1} g(x) f(x, t) d x, 0 \leq t \leq T .
\end{aligned}
$$


Proof. Let $\{u(x, t), p(t)\}$ be a classical solution of the problem (1)-(4). Differentiating both sides of (4) twice with respect to $t$, gives

$$
\begin{gathered}
\int_{0}^{1} g(x) u_{t}(x, t) d x=h^{\prime}(t), \\
\int_{0}^{1} g(x) u_{t t}(x, t) d x=h^{\prime \prime}(t), 0 \leq t \leq T .
\end{gathered}
$$

Multiplying both sides of (1) by a special function $g(x)$ and integrating gives, in turn,

$$
\begin{aligned}
& \frac{d^{2}}{d t^{2}} \int_{0}^{1} g(x) u(x, t) d x+\int_{0}^{1} g(x) u_{x x x x}(x, t) d x+\beta \int_{0}^{1} g(x) u_{x x}(x, t) d x \\
& \quad=p(t) \int_{0}^{1} g(x) u(x, t) d x+\int_{0}^{1} g(x) f(x, t) d x, 0 \leq t \leq T .
\end{aligned}
$$

Hence, taking into account (4) and (6), we conclude that condition (5) is satisfied.

Now, assume that $\{u(x, t), p(t)\}$ is the solution of problem (1)-(3), (5). Then from (5), taking into account (7), we have

$$
\begin{gathered}
\frac{d^{2}}{d t^{2}}\left(\int_{0}^{1} g(x) u(x, t) d x-h(t)\right) \\
=p(t)\left(\int_{0}^{1} g(x) u(x, t) d x-h(t)\right), 0 \leq t \leq T .
\end{gathered}
$$

By virtue of (2) and

$$
\int_{0}^{1} g(x) \varphi(x) d x=h(0), \int_{0}^{1} g(x) \psi(x) d x=h^{\prime}(0),
$$

we find

$$
\int_{0}^{1} g(x) u(x, 0) d x-h(0)=\int_{0}^{1} g(x) \varphi(x) d x-h(0)=0
$$




$$
\int_{0}^{1} g(x) u_{t}(0, t) d x-h^{\prime}(0)=\int_{0}^{1} g(x) \psi(x) d x-h^{\prime}(0)=0 .
$$

From (8), taking into account (9), it is clear that the condition (4) is also satisfied. The proof is complete.

\section{Classical Solvability of Inverse Boundary-value Problem}

We seek the first component $u(x, t)$ of classical solution $\{u(x, t), p(t)\}$ of the problem (1)-(3), (5) in the form

$$
u(x, t)=\sum_{k=1}^{\infty} u_{k}(t) \sin \lambda_{k} x, \lambda_{k}=k \pi,
$$

where

$$
u_{k}(t)=2 \int_{0}^{1} u(x, t) \sin \lambda_{k} x d x, k=1,2, \ldots
$$

Then applying the formal scheme of the Fourier method, from (1) and (2) we have

$$
\begin{gathered}
u_{k}^{\prime \prime}(t)+\left(\lambda_{k}^{4}-\beta \lambda_{k}^{2}\right) u_{k}(t)=F_{k}(t ; u, p), 0 \leq t \leq T ; k=1,2, \ldots, \\
u_{k}(0)=\varphi_{k}, u_{k}^{\prime}(0)=\psi_{k}, k=1,2, \ldots
\end{gathered}
$$

where

$$
\begin{gathered}
F_{k}(t ; u, p)=p(t) u_{k}(t)+f_{k}(t), f_{k}(t)=\int_{0}^{1} f(x, t) \sin \lambda_{k} x d x, \\
\varphi_{k}=2 \int_{0}^{1} \varphi(x) \sin \lambda_{k} x d x, \psi_{k}=2 \int_{0}^{1} \psi(x) \sin \lambda_{k} x d x, \quad k=1,2, \ldots
\end{gathered}
$$

Suppose that $0<\beta<\frac{\pi^{2}}{2}$. Solving the problem (11)-(12) gives

$$
\begin{gathered}
u_{k}(t)=\varphi_{k} \cos \beta_{k} t+\frac{1}{\beta_{k}} \psi_{k} \sin \beta_{k} t \\
+\frac{1}{\beta_{k}} \int_{0}^{t} F_{k}(\tau ; u, p) \sin \beta_{k}(t-\tau) d \tau, k=1,2, \ldots,
\end{gathered}
$$


where

$$
\beta_{k}=\sqrt{\lambda_{k}^{4}-\beta \lambda_{k}^{2}}
$$

In order to determine the first component of the solution of the problem (1)-(3), (5) we substitute $u_{k}(t)(k=1,2, \ldots)$ into (10) and obtain

$$
\begin{gathered}
u(x, t)=\sum_{k=1}^{\infty}\left\{\varphi_{k} \cos \beta_{k} t+\frac{1}{\beta_{k}} \psi_{k} \sin \beta_{k} t\right. \\
\left.+\frac{1}{\beta_{k}} \int_{0}^{t} F_{k}(\tau ; u, p) \sin \beta_{k}(t-\tau) d \tau\right\} \sin \lambda_{k} x .
\end{gathered}
$$

Now, from (5), taking into account (10), we have

$$
\begin{aligned}
& p(t)=[h(t)]^{-1}\left\{h^{\prime \prime}(t)-\int_{0}^{1} g(x) f(x, t) d x\right. \\
& \left.+\sum_{k=1}^{\infty}\left(\lambda_{k}^{4}-\beta \lambda_{k}^{2}\right) u_{k}(t) \int_{0}^{1} g(x) \sin \lambda_{k} x d x\right\} .
\end{aligned}
$$

In this way to obtain the equation for the second component of the solution to the problem (1) - (3), (5) we substitute expression (13) into (17) and get

$$
\begin{gathered}
p(t)=[h(t)]^{-1}\left\{h^{\prime \prime}(t)-\int_{0}^{1} g(x) f(x, t) d x\right. \\
+\sum_{k=1}^{\infty}\left(\lambda_{k}^{4}-\beta \lambda_{k}^{2}\right)\left[\varphi_{k} \cos \beta_{k} t+\frac{1}{\beta_{k}} \psi_{k} \sin \beta_{k} t\right. \\
\left.\left.+\frac{1}{\beta_{k}} \int_{0}^{t} F_{k}(\tau ; u, p) \sin \beta_{k}(t-\tau) d \tau\right] \int_{0}^{1} g(x) \sin \lambda_{k} x d x\right\} .
\end{gathered}
$$

Thus, finding the solution of problem (1) - (3), (5) is reduced to the finding solution of system (14), (16) with respect to unknown functions $u(x, t)$ and $p(t)$.

The following lemma plays an important role in studying the uniqueness of the solution to problem (1)-(3), (5).

Lemma 3. If $\{u(x, t), p(t)\}$ is a solution of (1)-(3), (5), then the functions

$$
u_{k}(t)=2 \int_{0}^{1} u(x, t) \sin \lambda_{k} x d x, k=1,2, \ldots,
$$

satisfy the system (13) on the interval $[0, T]$. 
Proof. Let $\{u(x, t), p(t)\}$ be any solution to problem (1)-(3), (5). Multiplying both sides of the Eq. (1) by a function $2 \sin \lambda_{k} x(k=1,2, \ldots)$, integrating by parts, and using the relations

$$
\begin{gathered}
2 \int_{0}^{1} u_{t t}(x, t) \sin \lambda_{k} x d x \\
=\frac{d^{2}}{d t^{2}}\left(2 \int_{0}^{1} u(x, t) \sin \lambda_{k} x d x\right)=u_{k}^{\prime \prime}(t), \quad k=1,2, \ldots, \\
=-\lambda_{k}^{2}\left(2 \int_{0}^{1} u(x, t) \sin \lambda_{k} x d x\right)=-\lambda_{k}^{2} u_{k}(t), k=1,2, \ldots, \\
=\lambda_{k}^{4}\left(2 \int_{0}^{1} u(x, t) \cos \lambda_{k} x d x\right)=\lambda_{k}^{4} u_{k}(t), k=1,2, \ldots,
\end{gathered}
$$

we deduce that satisfies (11).

Similarly, from (2) we obtain that condition (12) is satisfied. Thus, the system of functions $u_{k}(t)(k=1,2, \ldots)$ is a solution to problem (11), (12). From this it follows directly that the functions $u_{k}(t)(k=1,2, \ldots)$ satisfy the system (13) on $[0, T]$. The lemma is proved.

Obviously, if $u_{k}(t)=2 \int_{0}^{1} u(x, t) \sin \lambda_{k} x d x, k=1,2, \ldots$ is a solution to system (13), then the pair $\{u(x, t), p(t)\}$ of functions $u(x, t)=\sum_{k=1}^{\infty} u_{k}(t) \sin \lambda_{k} x$ and $p(t)$ is also a solution to system (14), (16).

It follows from Lemma 3 that

Remark 4. Suppose that system (14) and (16) has a unique solution. Then the problem (1)-(3), (5), can't have more than one solution, i.e. if problem (1)-(3), (7) has a solution, then it is unique. 
Now, to study problem (1)-(3), (5), we consider the following spaces.

Denote by $B_{2, T}^{5}$ a set of all functions of the form

$$
u(x, t)=\sum_{k=1}^{\infty} u_{k}(t) \sin \lambda_{k} x, \lambda_{k}=\pi k,
$$

considered in $D_{T}$ where each of the function $u_{k}(t)(k=1,2, \ldots)$ is continuous on $[0, T]$ and

$$
J_{T}(u) \equiv\left(\sum_{k=1}^{\infty}\left(\lambda_{k}^{2}\left\|u_{1}(t)\right\|_{C[0, T]}\right)^{2}\right)^{\frac{1}{2}}<+\infty .
$$

The norm in this set is defined as follows

$$
\|u(x, t)\|_{B_{2, T}^{5}}=J(u) .
$$

Next we denote by $E_{T}^{5}$ the space of vector-functions $z(x, t)=\{u(x, t), p(t)\}$, which $u(x, t) \in B_{2, T}^{5}, p(t) \in C[0, T]$.

The norm in the set $E_{T}^{5}$ will be

$$
\|z(x, t)\|_{E_{T}^{5}}=\|u(x, t)\|_{B_{2, T}^{5}}+\|p(t)\|_{C[0, T]} .
$$

It is known that $B_{2, T}^{5}$ and $E_{T}^{5}$ are the Banach spaces [21].

We now consider the operator

$$
\Phi(u, p)=\left\{\Phi_{1}(u, p), \Phi_{2}(u, p)\right\},
$$

in the space $E_{T}^{5}$, where

$$
\Phi_{1}(u, p)=\tilde{u}(x, t) \equiv \sum_{k=1}^{\infty} \tilde{u}_{k}(t) \sin \lambda_{k} x, \Phi_{2}(u, p)=\tilde{p}(t),
$$

and the functions $\tilde{u}_{k}(t)(k=1,2, \ldots)$ and $\tilde{p}(t)$ are equal to the right-hand sides of (13) and (16), respectively.

Then we find

$$
\begin{gathered}
\left(\sum_{k=1}^{\infty}\left(\lambda_{k}^{5}\left\|\tilde{u}_{k}(t)\right\|_{C[0, T]}\right)^{2}\right)^{\frac{1}{2}} \leq 2\left(\sum_{k=1}^{\infty}\left(\lambda_{k}^{5}\left|\varphi_{k}\right|\right)^{2}\right)^{\frac{1}{2}} \\
+\left(\sum_{k=1}^{\infty}\left(\lambda_{k}^{3}\left|\psi_{k}\right|\right)^{2}\right)^{\frac{1}{2}}+\sqrt{T}\left(\int_{0}^{T} \sum_{k=1}^{\infty}\left(\lambda_{k}^{3}\left|f_{k}(\tau)\right|\right)^{2} d \tau\right)^{\frac{1}{2}}
\end{gathered}
$$




$$
\begin{gathered}
+T\|p(t)\|_{C[0, T]}\left(\sum_{k=1}^{\infty}\left(\lambda_{k}^{5}\left\|u_{k}(t)\right\|_{C[0, T]}\right)^{2}\right)^{\frac{1}{2}} \\
\|\tilde{p}(t)\|_{C[0, T]} \leq\left\|[h(t)]^{-1}\right\|_{C[0, T]}\left\{\left\|h^{\prime \prime}(t)-\int_{0}^{1} g(x) f(x, t) d x\right\|_{C[0, T]}\right. \\
+\left(\sum_{k=1}^{\infty} \lambda_{k}^{-2}\right)^{\frac{1}{2}}(1+\beta)\left[\left(\sum_{k=1}^{\infty}\left(\lambda_{k}^{5}\left|\varphi_{k}\right|\right)^{2}\right)^{\frac{1}{2}}\right. \\
+\left(\sum_{k=1}^{\infty}\left(\lambda_{k}^{3}\left|\psi_{i k}\right|\right)^{2}\right)^{\frac{1}{2}}+\sqrt{T}\left(\int_{0}^{T} \sum_{k=1}^{\infty}\left(\lambda_{k}^{3}\left|f_{k}(\tau)\right|\right)^{2} d \tau\right)^{\frac{1}{2}} \\
+T\|p(t)\|_{C[0, T]}\left(\sum_{k=1}^{\infty}\left(\lambda_{k}^{5}\left\|u_{k}(t)\right\|_{C[0, T]}\right)^{2}\right)^{\frac{1}{2}}\|g(x)\|_{L_{2}(0,1)}
\end{gathered}
$$

Suppose that the data of problem (1)-(3), (5) satisfy the conditions

$\left(A_{1}\right) \varphi(x) \in C^{4}[0,1], \varphi^{(5)}(x) \in L_{2}(0,1)$, $\varphi(0)=\varphi(1)=\varphi^{\prime \prime}(0)=\varphi^{\prime \prime}(1)=\varphi^{(4)}(0)=\varphi^{(4)}(1)=0 ;$

$\left(A_{2}\right) \varphi(x) \in C^{4}[0,1], \psi^{\prime \prime \prime}(x) \in L_{2}(0,1)$, $\psi(0)=\psi(1)=\psi^{\prime \prime}(0)=\psi^{\prime \prime}(1)=0$

$\left(A_{3}\right) f(x, t), f_{x}(x, t), f_{x x}(x, t) \in C\left(D_{T}\right), f_{x x x}(x, t) \in L_{2}\left(D_{T}\right)$, and $f(0, t)=$ $f(1, t)=f_{x x}(0, t)=f_{x x}(1, t)=0,0 \leq t \leq T$;

$\left(A_{4}\right) 0<\beta<\frac{\pi^{2}}{2}, g(x) \in L_{2}(0,1), h(t) \in C^{2}[0, T]$, $h(t) \neq 0,0 \leq t \leq T$.

Then from relations (17) and (18), correspondingly we have

$$
\begin{gathered}
\left(\sum_{k=1}^{\infty}\left(\lambda_{k}^{5}\left\|\tilde{u}_{k}(t)\right\|_{C[0, T]}\right)^{2}\right)^{\frac{1}{2}} \\
\leq 2\left\|\varphi^{(5)}(x)\right\|_{L_{2}(0,1)}+\left\|\psi^{\prime \prime \prime}(x)\right\|_{L_{2}(0,1)}+\sqrt{T}\left\|f_{x x x}(x, t)\right\|_{L_{2}\left(D_{T}\right)} \\
+T\|p(t)\|_{C[0, T]}\left(\sum_{k=1}^{\infty}\left(\lambda_{k}^{5}\left\|u_{k}(t)\right\|_{C[0, T]}\right)^{2}\right)^{\frac{1}{2}},
\end{gathered}
$$




$$
\begin{gathered}
\|\tilde{p}(t)\|_{C[0, T]} \leq\left\|[h(t)]^{-1}\right\|_{C[0, T]}\left\{\left\|h^{\prime \prime}(t)-\int_{0}^{1} g(x) f(x, t) d x\right\|_{C[0, T]}\right. \\
+\left(\sum_{k=1}^{\infty} \lambda_{k}^{-2}\right)^{\frac{1}{2}}(1+\beta)\left[\left\|\varphi^{(5)}(x)\right\|_{L_{2}(0,1)}+\left\|\psi^{\prime \prime \prime}(x)\right\|_{L_{2}(0,1)}\right. \\
+\sqrt{T}\left\|f_{x x x}(x, t)\right\|_{L_{2}\left(D_{T}\right)}+T\|p(t)\|_{C[0, T]} \\
\left.\left.\times\left(\sum_{k=1}^{\infty}\left(\lambda_{k}^{5}\left\|u_{k}(t)\right\|_{C[0, T]}\right)^{2}\right)^{\frac{1}{2}}\right]\|g(x)\|_{L_{2}(0,1)}\right\} .
\end{gathered}
$$

We denote by

$$
\begin{gathered}
A_{1}(T)=2\left\|\varphi^{(5)}(x)\right\|_{L_{2}(0,1)}+\left\|\psi^{\prime \prime \prime}(x)\right\|_{L_{2}(0,1)}+\sqrt{T}\left\|f_{x x x}(x, t)\right\|_{L_{2}\left(D_{T}\right)}, \\
B_{1}(T)=T \\
A_{2}(T)=\left\|[h(t)]^{-1}\right\|_{C[0, T]}\left\{\left\|h^{\prime \prime}(t)-\int_{0}^{1} g(x) f(x, t) d x\right\|_{C[0, T]}\right. \\
+\left(\sum_{k=1}^{\infty} \lambda_{k}^{-2}\right)^{\frac{1}{2}}(1+\beta)\left[\left\|\varphi^{(5)}(x)\right\|_{L_{2}(0,1)}\right. \\
\left.\left.+\left\|\psi^{\prime \prime \prime}(x)\right\|_{L_{2}(0,1)}+\sqrt{T}\left\|f_{x x x}(x, t)\right\|_{L_{2}\left(D_{T}\right)}\right]\|g(x)\|_{L_{2}(0,1)}\right\} \\
B_{2}(T)=\left\|[h(t)]^{-1}\right\|_{C[0, T]}\left(\sum_{k=1}^{\infty} \lambda_{k}^{-2}\right)^{\frac{1}{2}}(1+\beta) T\|g(x)\|_{L_{2}(0,1)},
\end{gathered}
$$

and rewrite (19), (20) as

$$
\begin{gathered}
\|\tilde{u}(x, t)\|_{B_{2, T}^{5}} \leq A_{1}(T)+B_{1}(T)\|p(t)\|_{C[0, T]}\|u(x, t)\|_{B_{2, T}^{5}}, \\
\|\tilde{p}(t)\|_{C[0, T]} \leq A_{2}(T)+B_{2}(T)\|p(t)\|_{C[0, T]}\|u(x, t)\|_{B_{2, T}^{5}} .
\end{gathered}
$$

From the inequalities (21) and (22), we conclude

$$
\|\tilde{u}(x, t)\|_{B_{2, T}^{5}}+\|\tilde{p}(t)\|_{C[0, T]}
$$




$$
\leq A(T)+B(T)\|p(t)\|_{C[0, T]}\|u(x, t)\|_{B_{2, T}^{5}},
$$

where

$$
A(T)=A_{1}(T)+A_{2}(T), B(T)=B_{1}(T)+B_{2}(T) .
$$

Thus, the following assertion is valid.

Theorem 5. If conditions $\left(A_{1}\right)-\left(A_{4}\right)$ and

$$
B(T)(A(T)+2)^{2}<1,
$$

hold, then problem (1)-(3), (5) has a unique solution in the ball $K=K_{R}\left(\|z\|_{E_{T}^{5}} \leq\right.$ $R=A(T)+2)$ of the space $E_{T}^{5}$.

Proof. In the space $E_{T}^{5}$, consider the equation

$$
z=\Phi z
$$

where $z=\{u, p\}$, the components $\Phi_{i}(u, p), i=1,2$, of operator $\Phi(u, p)$, defined by the right sides of equations (14) and (16), respectively.

Now, consider the operator $\Phi(u, p)$ in the ball $K=K_{R}$ of the space $E_{T}^{5}$. Similarly to (23), we obtain that for any $z=\{u, p\}, z_{1}=\left\{u_{1}, p_{1}\right\}$, and $z_{2}=$ $\left\{u_{2}, p_{2}\right\} \in K_{R}$ the following inequalities hold:

$$
\begin{gathered}
\|\Phi z\|_{E_{T}^{5}} \leq A(T)+B(T)\|p(t)\|_{C[0, T]}\|u(x, t)\|_{B_{2, T}^{5}}, \\
\left\|\Phi z_{1}-\Phi z_{2}\right\|_{E_{T}^{5}} \leq B(T) R \\
\times\left(\left\|p_{1}(t)-p_{2}(t)\right\|_{C[0, T]}+\left\|u_{1}(x, t)-u_{2}(x, t)\right\|_{B_{2, T}^{5}}\right) .
\end{gathered}
$$

Then by (24), from (14) and (16) it follows that the operator $\Phi$ acts in the ball $K=K_{R}$, and satisfy the conditions of the contraction mapping principle. Therefore the operator $\Phi$ has a unique fixed point $\{u, p\}$ in the ball $K=K_{R}$, which is a solution of equation (25), i.e. the pair $\{u, p\}$ is the unique solution of the systems (14), (16) in $K=K_{R}$.

Then the function $u(x, t)$ as an element of space $B_{2, T}^{5}$ is continuous and has continuous derivatives $u_{x}(x, t), u_{x x}(x, t), u_{x x x}(x, t)$, and $u_{x x x x}(x, t)$ in $D_{T}$.

From (9) it is easy to see that

$$
\left(\sum_{k=1}^{\infty}\left(\lambda_{k}\left\|u_{i k}^{\prime \prime}(t)\right\|_{C[0, T]}\right)^{2}\right)^{\frac{1}{2}} \leq(1+\beta)
$$




$$
\times\left(\sum_{k=1}^{\infty}\left(\lambda_{k}^{5}\left\|u_{k}(t)\right\|_{C[0, T]}\right)^{2}\right)^{\frac{1}{2}}+\|\| f_{x}(x, t)+p(t) u_{x}(x, t)\left\|_{C[0, T]}\right\|_{L_{2}(0,1)} .
$$

Hence, we conclude that the function $u_{t t}(x, t)$ is continuous in the domain $D_{T}$.

Further, it is easy to verify that equation (1), and conditions (2), (3), and (5) are satisfied in the usual sense. Consequently, $\{u(x, t), p(t)\}$ is a solution of (1)-(3), (5), and by Lemma 3 it is unique in the ball $K=K_{R}$. The proof is complete.

From Theorem 2 and Theorem 5, it implies the unique solvability of the original problem (1)-(4).

In summary, we conclude the following result.

Theorem 6. Suppose that all assumptions of Theorem 5, and

$$
\int_{0}^{1} g(x) \varphi(x) d x=h(0), \int_{0}^{1} g(x) \psi(x) d x=h^{\prime}(0)
$$

hold. Then problem (1)-(4) has a unique classical solution in the ball $K=$ $K_{R}\left(\|z\|_{E_{T}^{5}} \leq R=A(T)+2\right)$.

Acknowledgements. The authors are sincerely grateful to the editors and anonymous reviewers for carefully reading the manuscript, as well as for their valuable comments and suggestions that helped improve this article.

\section{References}

[1] A.Ya. Akhundov, A nonlinear parabolic inverse coefficient problem, Transactions Issue Mathematics, Series of Physical-Technical and Mathematical Science, NAS Azerbaijan, 4 (2002), 19-24.

[2] E.I. Azizbayov, Y.T. Mehraliyev, A boundary value problem for the equation of motion of a homogeneous bar with periodic conditions, American Journal of Applied Mathematics and Statistics, 3 (2015), 252-256.

[3] E.I. Azizbayov, Y.T. Mehraliyev, On a nonlocal inverse problem with the integral overdetermination condition for a parabolic equation of second order, International Journal of Pure and Applied Mathematics, 116 (2017), 601-616. 
[4] E. Azizbayov, The nonlocal inverse problem of the identification of the lowest coefficient and the right-hand side in a second-order parabolic equation with integral conditions, Boundary Value Problems, 2019 (2019), 1-9.

[5] B.S. Bardin, S.D. Furta, Local Theory of Existence of the Periodic Wave Movements of an Infinite Beam on not Linearly Elastic Basis, In book: Actual Problems Classical and Heavenly Mechanics, Moscow (1998) (in Russian).

[6] Yu.Ya. Belov, Inverse Problems of Mathematical Physics, Inverse and Illposed Problems Series, VSP (2002).

[7] G. Eskin, Inverse problems for general second order hyperbolic equations with time-dependent coefficients, Bulletin of Mathematical Sciences, 7 (2017), 247-307.

[8] T. Goy, M. Negrych, and I. Savka, On nonlocal boundary value problem for the equation of motion of a homogeneous elastic beam with pinned-pinned ends, Carpathian Mathematical Publications, 10 (2018), 105-113.

[9] A.D. Iskenderov, T.B. Gardashov, Simulation solutions and inverse problems for quasi-linear parabolic equations, Differential Equations, 26 (1990), 836-840.

[10] M.I. Ivanchov, Inverse Problem for Equations of Parabolic Type, Mathematical Studies, Lviv (2003).

[11] V.K. Ivanov, On linear problems which are not well-posed, Doklady Akademii Nauk SSSR, 145 (1962), 270-272 (in Russian).

[12] Yu.A. Izyumov, V.N. Syromyatnikov, Phase Transitions and Crystal Symmetry, Springer (1990).

[13] J. Janno, A. Šeletski, Reconstruction of coefficients of higher order nonlinear wave equations by measuring solitary waves, Wave Motion, 52 (2015), $15-25$.

[14] S.I. Kabanikhin, Inverse and Ill-posed Problems, Theory and Applications, De Gruyter, Berlin (2012).

[15] D.V. Kostin, Analysis scheme for bimodal deflections of a weakly inhomogeneous elastic beam, Doklady Mathematics, 418 (2008), 46-50. 
[16] M.M. Lavrent'ev, V.G. Vasil'ev, and V.G. Romanov, Multidimensional Inverse Problems for Differential Equations, Lecture Notes in Mathematics, Berlin (1970).

[17] Y.T. Mehraliyev, On an inverse boundary-value problem for a second order elliptic equation with integral condition, Visnyk of the Lviv University, Ser. Mechanics and Mathematics, 77 (2012), 145-156.

[18] Yu.O. Mitropolsky, B.I. Moseenkov, Study of Oscillations in Systems with Distributed Parameters, Publishing House of Kyiv University, Kyiv (1961) (in Ukrainian).

[19] A.I. Prilepko, D.G. Orlovsky, and I.A. Vasin, Methods for Solving Inverse Problems in Mathematical Physics, Marcel Dekker, New York (2000).

[20] S.G. Pyatkov, On some classes of inverse problems for parabolic equations, Journal of Inverse and Ill-posed Problems, 18 (2011), 917-934.

[21] I. Tekin, Y.T. Mehraliyev, and M.I. Ismailov, Existence and uniqueness of an inverse problem for nonlinear Klein-Gordon equation, Mathematical Methods in the Applied Sciences, 42 (2019), 3739-3753.

[22] J.M.T. Thompson, A General Theory of Elastic Stability, John Wiley \& Sons (1973).

[23] A.N. Tikhonov, On stability of inverse problems, Doklady Akademii Nauk SSSR, 39 (1943), 195-198 (in Russian).

[24] V.Z. Vlasov, N.N. Leont'ev, Beams, Plates and Covers on the Elastic Basis, Moscow (1960). 